\title{
Some Chemical Aspects of Human Brain Development. I. Neutral Glycosphingolipids, Sulfatides, and Sphingomyelin
}

\author{
Carmen Conde, Manuela Martinez, and Angel Ballabriga ${ }^{[18]}$ \\ Autonomous University, School of Medicine, Children's Hospital of the \\ "Seguridad Social," Barcelona, Spain
}

\begin{abstract}
Extract
Neutral glycosphingolipids and sulfatides, as well as sphingomyelin, were studied in the brain of 34 newborn infants with gestational ages ranging from 22 to 45 weeks. The analyses were carried out in homogenates of whole cerebrum. The infants were considered to be free of any important cause of cerebral damage. Neutral glycosphingolipids and sulfatides were expressed by their content in hexose. Sphingomyelin was separated from other lipids by column chromatography and alkaline hydrolysis, and was expressed by its phosphorus content. The figures were plotted versus gestational ages. The index, brain weight/body weight per cent, was plotted against gestational ages in order to compare the increase of brain weight with the deposition of lipids.
\end{abstract}

\section{Speculation}

The 3rd trimester of human gestation is a period during which the increase in brain weight and developmental processes proceed at a very high velocity. The study of a wide series of human brains obtained under the same conditions from newborn infants with a narrow range of gestational ages makes it possible to follow the biochemical changes from an early stage of maturation. This allows minimal variations to be detected in a period of maximal sensitivity.

Sphingomyelin and neutral glycosphingolipids and sulfatides were chosen for the purpose of tracing the way in which the developmental changes proceed. The chronologic relation between biochemical changes and brain weight increase was considered also.

\section{Introduction}

The 3rd trimester of human gestation is the initial period of "growth spurt." During the last few years, a number of papers based on animal experimentation $[1,2,4,7,15]$ have been published, allowing certain periods of cellular multiplication and myelination in different species of animals to be established.
Dobbing and Sands [6] measured the content of DNA in the human brain and described cellular multiplication as a growth phenomenon consisting of two periods of maximum activity, the first ranging between the 15th and the 20th week of gestational age, and the second beginning in the 25th week and characterized by a sharp, linear increase up to the 45 th week. The first period of high activity is due to the proliferation 
of neuroblasts, and the second to glial proliferation. The same phenomena are produced in the cerebellum and cerebral stem, although with chronologic differences.

Winick [15] also studied a group of 31 human brains with ages ranging between 13 weeks of gestational age and a 13 months of postnatal age. He determined the content of DNA, RNA, and protein, and came to the conclusion that cellular multiplication ends at the age of 5 months. Dobbing [5] objected to this opinion and stated that the human brain growth spurt must be sonsidered to extend from midpreg. nancy to the 2 nd postnatal year.

To follow the myelination from its earliest stages constitutes a very difficult methodologic problem. The myelin lipids, cerebrosides, and sulfatides are found in a very low concentration in the immature brain. With the methods of isolation used by the workers interested in this field, other sphingolipids are extracted together with cerebrosides and sulfatides not related to myelination. Only with maturation is this lipid fraction enriched in myelin lipids, namely sulfatides and cerebrosides Svennerholm studied the changes of such compounds during development $[12,14]$.

\section{Materials and Methods}

Thirty-four brains, selected from 600 brains of newborn infants with different gestational ages, who died in our clinic during the last 2 years, were considered suitable for this study. Those cases in which the major cause of death could be related to subarachnoid hemorrhage, brain damage, malformations of any kind, or neonatal asphyxia which required deep resucitation were excluded. The major causes of death were pulmonary hemorrhage, immaturity, hyaline membrane disease, and congenital pneumonia. In each case the nutritional state of the mother was checked to exclude cases with maternal malnutrition.

Gestational age was determined by physical examination as well as by maternal history, reckoning from the 1st day of the last menstrual period. The infants were classified as appropriate for gestational age in accordance with Lubchenko's grid. The analyses were carried out in whole cerebrum. After extracting meninges and blood, the hemisphere was weighted and homogenized in an electric mixer [17]. The homogenate was kept at $-20^{\circ}$ and wrapped in aluminum foil and in air-tight, closed polyethylene bags. The lipids were extracted by Svennerholm's procedure [13] in order to remove the gangliosides as completely as possible.

\section{Biochemical Methods}

\section{Neutral Glycosphingolipids and Sulfatides}

The lipid extracts (lower phase) were freed from most of the phospholipids by chromatography on Floorisil, according to the procedure described by Sven. nerholm [12]. Portions of the lipid extract containing as much as $300 \mathrm{mg}$ brain homogenate were used for the determination of the glycosphingolipids because of their low concentration in the samples of immature brain. Total hexose was determined by the orcinol method of Svennerholm [11] after hydrolysis with chloroform-methanol-hydrochloric acid following the procedure given by Radin et al. [8]. The results were given as content in hexose per $100 \mathrm{mg}$ wet tissue. The fact that we were measuring a mixture of different compounds made it impossible to give the concentrations in micromoles or milligrams.

\section{Sphingomyelin}

The lower phase of the lipid extract was employed. Sphingomyelin was freed from other phospholipids by chromatography on alumina. The choline-containing phospholipids were eluted with chloroform-methanol $(1 / 1, v / v)$, and phosphorus was determined by the FiskeSubbaRow method after a mild alkaline hydrolysis. As for the characteristics of the lipid extracts, columns, and eluates, the methodology described by Svennerholm [12] was followed. The results were expressed in micrograms of phosphorus per $100 \mathrm{mg}$ wet tissue.

\section{Results and Discussion}

The concentration of neutral glycosphingolipids and sulfatides (Fig. 1) as well as sphingomyelin concentration (Fig. 2) were plotted versus gestational age. Both graphs indicate that there is a time during the last trimester of intrauterine life in which a biochemical change appears almost abruptly. The figures of both sphingomyelin and glycosphingolipids constitute a nearly homogeneous population in a first period which extends from the 22nd week of gestational age until a limit difficult to fix, between the 29th and the 31st weeks of gestational age. After the 32nd week a very clear increase appears in spite of the dispersion of values. This sharp increase extends until the 45th week, which is the limit of our study.

In the case of neutral glycosphingolipids and sulfatides, the profile of the graph may be considered to be a good approach to the process of myelination if we bear in mind that this lipid fraction is almost entirely 


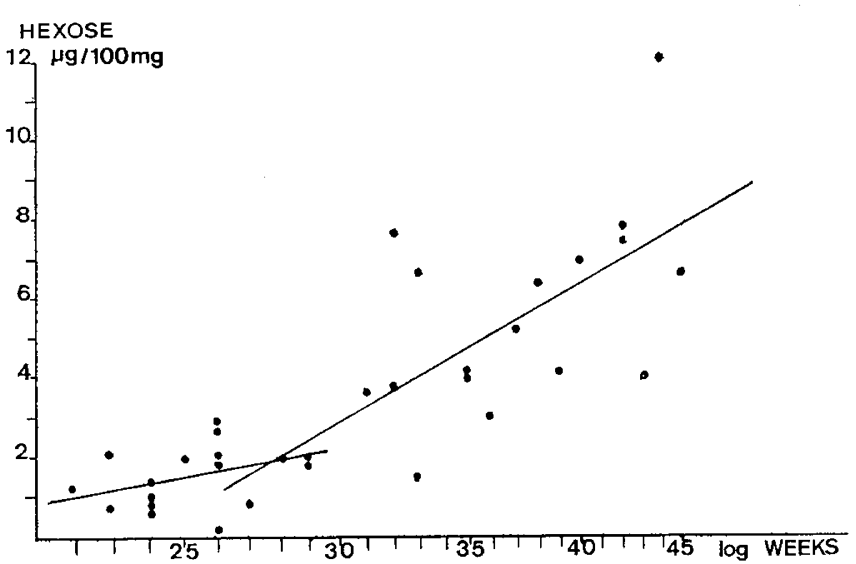

Fig. 1. Neutral glycosphingolipids plus sulfatides. The first regression line $(y=7.773 \log \mathrm{x}-9.357)$ has a low slope, and the increase is not statistically significant $(\mathrm{r}=0.352, P>0.1)$, whereas after the 29th week the increase is much faster $(y=27.645 \log$ $x-37.874)$ and at a 0.005 level of significance $(r=0.636)$.

constituted by cerebrosides and sulfatides in the cerebrum at term.

The change in rate of deposition of lipids in both cases is more apparent if concentrations are plotted against the $\log _{10}$ of gestational age as Rouser and Yamamoto suggest [9]. Two regression lines were traced, the first until the 29th week, and the second from the 29th to the 45th week. The slope for the first population was not only very slight but also without statistical significance. The increase was sharper in both cases for the second population and had a higher statistical significance.

Figure 3 indicates that the period of maximum increase in brain weight in relation to body weight has about the same duration as the period of homogeneous concentration of lipids in Figures 1 and 2. The cerebral tissue seems to change towards a rapid maturation when the brain weight begins to increase more slowly in relation to body weight.

Rouser et al. [10] investigated the changes with age of the lipid composition of whole cerebrum. They studied the interrelation of the lipid classes during development in order to provide information about the lipid compounds related to maturation. They defined as substitution group $I$ the changes with age consisting of an increase in cerebrosides, sulfatides, and sphingomyelin concentration, accompanied by a decrease in phosphtidyl ethanolamine and phosphatidyl choline. The authors described such changes in representative samples of whole brain. They had failed to find them in earlier studies using white and gray matter.

Svennerholm and Vanier [14] did not accept the assumption of the study of whole brain as more repre- sentative, and separated cerebral cortex and white matter in their study of the lipid composition in fetal and infant brain. They reported an increase in the concentration of sulfatides, cerebrosides, and sphingomyelin, and a decrease of phosphatidyl choline in white matter and cerebral cortex, with maturation, during the postnatal period. No figures were given for the concentration of cerebrosides and sulfatides in prenatal life, nor was the white matter studied in that period.

In the present paper, the suggestions of Rouser $e t$ al. [10] have been followed and the whole cerebrum has

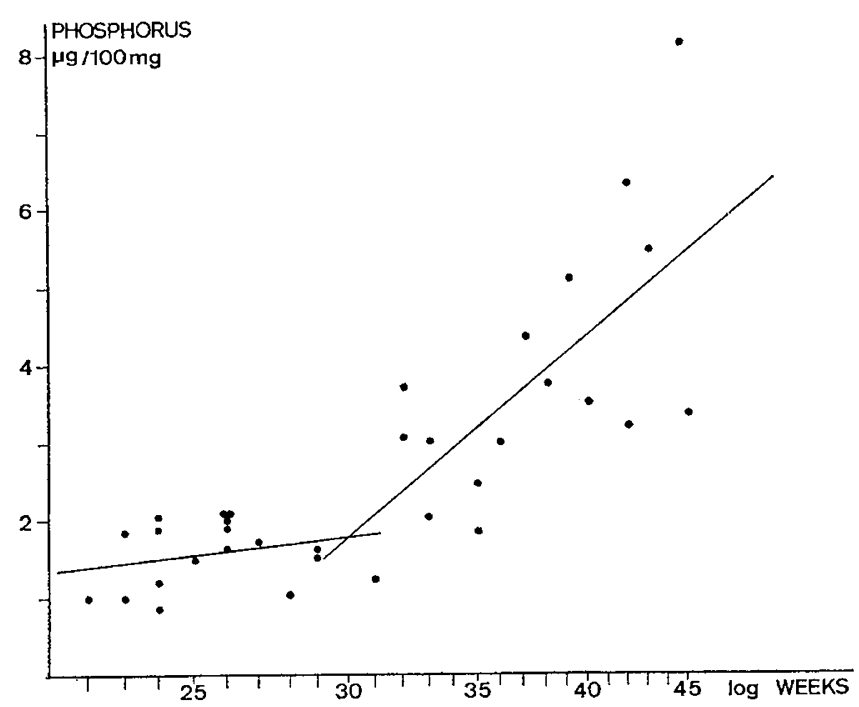

Fig. 2. Sphingomyelin. Until the 29 th week of gestational age the increase of sphingomyelin is negligible $(y=2.871 \log x-2.440)$ and without statistical significance $(\mathrm{r}=0.238, P>0.1)$. Afterward the slope of the increase is pronounced $(y=21.254 \log x-$ $29.625)$ and highly significant $(\mathrm{r}=0.733, P<0.001)$.

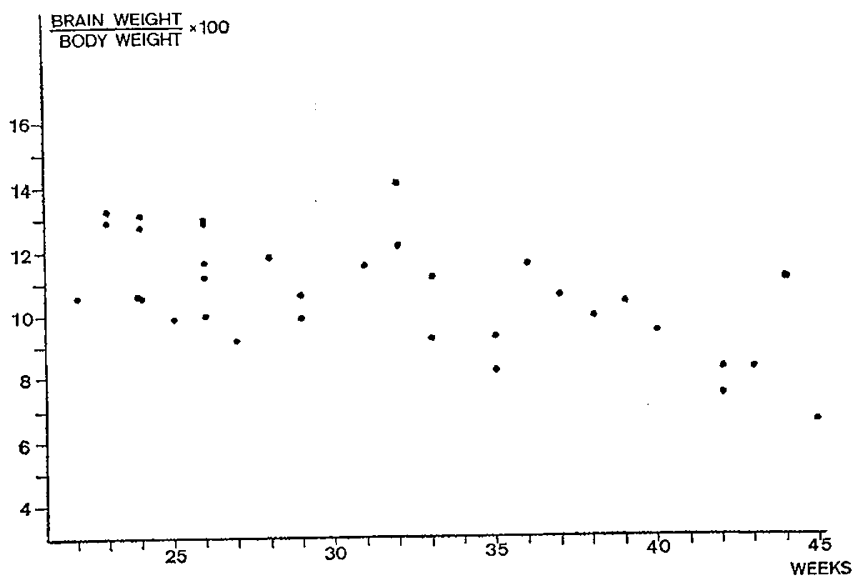

Fig. 3. (Brain weight/body weight) $\times 100$ index. The percentage of brain weight in relation to body weight decreases markedly after the 32nd week of gestational age. 
been used because of methodologic difficulties in the separation of gray and white matter, which makes it impossible to obtain tissue in sufficient amounts for the quantification of glycosphingolipids.

\section{Summary}

Neutral glycosphingolipids, sulfatides, and sphingomyelin were studied in brains of newborn infants from the 22nd to the 45th week of gestational age. The cases belonging to the period between the 22nd and the 29 th week seem to constitute an homogeneous population without any significant variation in biochemical maturation. A very sharp increase in the concentration of these compounds was seen from the 32nd week onwards.

\section{References and Notes}

1. Cuzner, M. L., and Davison, A. N.: The lipid composition of rat brain myelin and subcellular fractions during development. Biochem. J., 106: 29 (1968).

2. Davison, A. N., Cuzner, M. L., Banick, N. L., ANd Oxberry, J.: Myelinogenesis in rat brain. Nature, 212: 1373 (1966).

3. Dickerson, J. W. T., Merat, A., and Widdowson, E. M.: Intrauterine growth retardation in the pig. Biol. Neonate, 19: 354 (1971).

4. Dobbing, J.: In A. N. Davison and J. Dobbing: Applied Neurochemistry (Blackwell, Oxford, 1968).

5. Dobbing, J.: Letter to the Editor. Lancet, $i$ : 48 (1973).
6. Dobbing, J., and SAnds, J.: Timing of neuroblast multiplication in developing human brain. Nature (226), 5246: 639 (1970).

7. Galli, C., and CECCONI, R. D.: Lipid changes in rat brain during maturation. Lipids, 2: 76 (1967).

8. Radin, N. S., BRown, J. R., ANd Lavin, F. B.: The preparative isolation of cerebrosides. J. Biol. Chem., 219: 977 (1956).

9. Rouser, G., And Yamamoto, A.: Curvilinear regression course of human brain lipid composition. Lipids, 3(3): 284 (1968).

10. Rouser, G., Yamamoto, A., and Kritchevsky, G.: In: R. Paoletti and A. Davison: Chemistry and Brain Development, p. 91 (Plenum Press, New York, 1971).

11. Svennerholm, L.: The quantitative estimation of cerebrosides in nervous system. J. Neurochem., 1: 42 (1956).

12. Svennerholm, L.: The distribution of lipids in the human nervous system. J. Neurochemistry, 2: 839 (1964).

13. SVENNERHOLM, L.: Distribution and fatty acids composition of phosphoglycerides in normal human brain. J. Lipid Res., 9: 570 (1968).

14. Svennerholm, L., and Vanier, M. T.: The distribution of lipids in human nervous system. II. Lipid composition of human foetal and infant brain. Brain Res., 47: 457 (1972).

15. Winick, M.: Changes in nucleic acid and protein content of the human brain during growth. Pediat. Res., 2: 352 (1968).

16. Winick, M., ANd Noble, A.: Quantitative changes in DNA, RNA and protein during prenatal and postnatal growth in the rat. Develop. Biol., 12: 451 (1965).

17. Ivan Sorvall, Inc., Norwalk, Conn.

18. Requests for reprints should be addressed to: A. Ballabriga, M.D., Children's Hospital of the "Seguridad Social," Paseo del Valle del Hebron s/n, Barcelona, Spain.

19. Accepted for publication September 26, 1973. 